\title{
Isolation of Naturally Induced T-regulatory Cells in Gingival Tissues of Healthy Human Subjects and Subjects with Gingivitis and Chronic Periodontitis
}

\author{
Devi Arul ${ }^{1}$, Suresh Rao ${ }^{1}$ \\ 1. Periodontics, Sri Ramachandra Medical College and Research Institute, Chennai, IND
}

Corresponding author: Devi Arul, devis.dr@gmail.com

\begin{abstract}
Background: The immune mechanism depends on CD4+ T cells for its regular function, and altered $\mathrm{T}$ cell function leads to microbial disease progression.

Aim: The present study aimed to determine the role of naturally induced T-regulatory (nTreg) cells (CD4+ CD25+ Fox P3+) in periodontal disease pathogenesis.

Materials and methods: A total of 30 patients attending the out-patient clinic of the Department of Periodontology and Implantology, Faculty of Dental Sciences, Sri Ramachandra University (SRU), Chennai, India were recruited for the study. They were categorized in three groups as healthy individuals, individuals with chronic gingivitis, and individuals with chronic periodontitis gingival tissues. nTreg (CD4+ CD25+ Fox $\mathrm{P3}+$ ) cells were isolated using flow cytometry. Different conjugated, isolated cells were then gated in the order of $\mathrm{CD} 4+, \mathrm{CD} 25+$, and Fox P3+ cells.
\end{abstract}

Results: The results of our study showed an increase in the proportions of Treg cells in individuals with chronic periodontitis compared to individuals with gingivitis and healthy individuals.

Conclusion: Further elucidation of cellular and molecular processes underlying Treg cells will help unravel the complexity behind periodontal disease pathogenesis besides paving the way in developing newer treatment strategies.

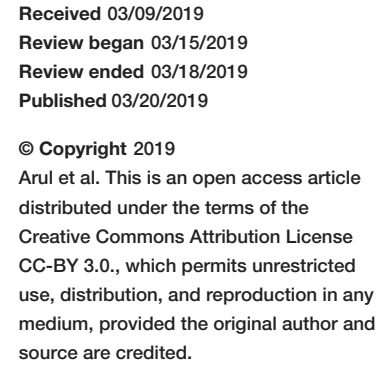

Categories: Miscellaneous, Allergy/Immunology

Keywords: regulatory t cells, ntreg, self tolerance, flow cytometry, chronic periodontitis

\section{Introduction}

Periodontal disease is a multifactorial disease where infection and its sequel, inflammation and immunity, play a critical role in disease pathogenesis [1]. Though the indispensable role of microorganisms leads to the initiation of disease, the progression and severity of the disease are due to the exaggerated host immune response. Periodontal disease is characterized by periods of exacerbation interspersed with periods of remission and is also regarded as a healing lesion. Available evidence suggests T-regulatory (Treg) cells as key anti-inflammatory cells that are critically involved in limiting the inflammatory response [2]. These cells are described as those $\mathrm{T}$ cells that have escaped deletion in the thymus and later become anergic or get suppressed by various mechanisms [3]. Treg cells can be defined as a group of T-cell population that functionally suppresses an immune response by influencing the activity of another cell type [4].

A major challenge in immunology and medicine was to determine the unresponsiveness of the adaptive immune system to self-antigens (that is, immunological self tolerance), which is now well established [5]. The immune mechanism has to deal with an array of inflammatory signals during long established infections like chronic periodontitis. There may be self control mechanisms where regulation of effector cells may take place. To assess this control mechanism, our present study was carried out to determine the presence of naturally induced Treg (nTreg) cells in states of gingival health, gingivitis, and chronic periodontitis.

\section{Materials And Methods}

\section{Study population}

This study was approved by the institutional ethics committee of Sri Ramachandra University, Chennai, India. A written informed consent was obtained from all the patients included in the study. The study comprised of three groups namely healthy individuals (group I; $\mathrm{n}=10$ ), chronic generalized gingivitis (group II; $\mathrm{n}=10$ ), and chronic periodontitis (group III; n=10) (Figure 1). 


\section{Cureus}

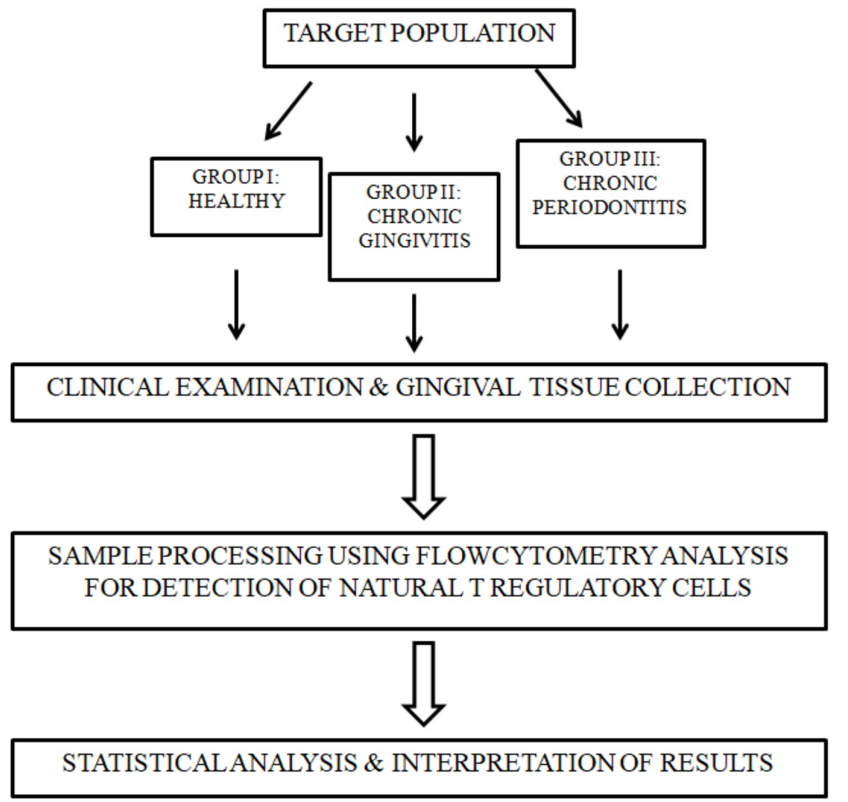

FIGURE 1: Selection of cases for the study.

Patients with clinically healthy gingiva with absence of bleeding on probing were included in group I. Patients with chronic generalized gingivitis with clinical signs of gingival inflammation with no evidence of clinical attachment loss or radiographic bone loss were included in group II. Patients with more than 20 teeth with clinical attachment loss >1-2 mm and radiographic evidence of bone loss were included in group III [6].

The exclusion criteria for all the groups were presence of any systemic disease, use of tobacco in any form, previous history of periodontal therapy, and those who had taken antibiotics or analgesics recently. Pregnant and lactating women were excluded from the study.

Healthy gingival tissues were obtained during crown lengthening procedures or therapeutic orthodontic extractions; chronic generalized gingivitis samples were obtained during gingivectomy procedures, and chronic generalized periodontitis tissue samples were obtained from hopeless teeth prior to extraction procedure.

\section{Reagents used and flow cytometry analysis}

The following markers that are specific for regulatory $\mathrm{T}$ cells were used in the analysis.

Fluorochrome conjugated monoclonal antibodies (Bd Biosciences, San Diego, CA, USA):

. 555348 PE-Cy 5 Mouse Anti-Human CD4

. 555432 PE Mouse Anti-Human CD25

. 561181 Alexa Flour 488 Mouse Anti-Human Fox P3

- Fox P3 buffer kit for fixing and permeabilization

o 51-9005451 (Fox P3 buffer A)

o 51-9005450 (Fox P3buffer B)

. 554656 BD Stain buffer (Fetal bovine Serum)

· Phosphate Buffered Saline pH 7.4 
All samples were thawed on ice and mononuclear cell suspensions were prepared using an ultrasonic processor with 100-200 $\mu$ l of phosphate buffered saline (PBS). The prepared cells were centrifuged at 1500 rpm for three minutes following which the supernatant was discarded. Then $200 \mu \mathrm{l}$ of PBS was added to the pellet and again centrifuged at $1500 \mathrm{rpm}$ for three minutes.

Thus a final cell pellet was obtained to which $100 \mu \mathrm{l}$ of PBS, $20 \mu \mathrm{L}$ of CD4 PECY5, $20 \mu \mathrm{l}$ of CD25PE, and $100 \mu \mathrm{l}$ of stain buffer were added. The samples were then incubated on ice for an hour in the dark following which they were again centrifuged. For intracellular staining, a final workable solution of Fox P3 buffer C was obtained by diluting Fox P3 buffer A and B according to the instructions of the manufacturer.

Following this, $1 \mathrm{ml}$ of Buffer ' $\mathrm{A}$ ' was added to the pellet and incubated for 10 minutes at room temperature. The samples were then washed with a stain buffer and underwent centrifugation. The obtained cell pellets were re-suspended in $500 \mu \mathrm{l}$ of buffer ' $\mathrm{C}$ ' and incubated for 30 minutes at room temperature. After a wash with $1 \mathrm{ml}$ of stain buffer, $100 \mu$ PBS and $5 \mu$ of Fox P3 Alexa Flour 488 were added to the cell pellets and incubated for 30 minutes at room temperature. The prepared samples were re-suspended in sheath fluid, following which they were transferred to a fluorescence-activated cell sorting (FACS) tube and used for cell acquisition and flow cytometry analysis (Figure 2).

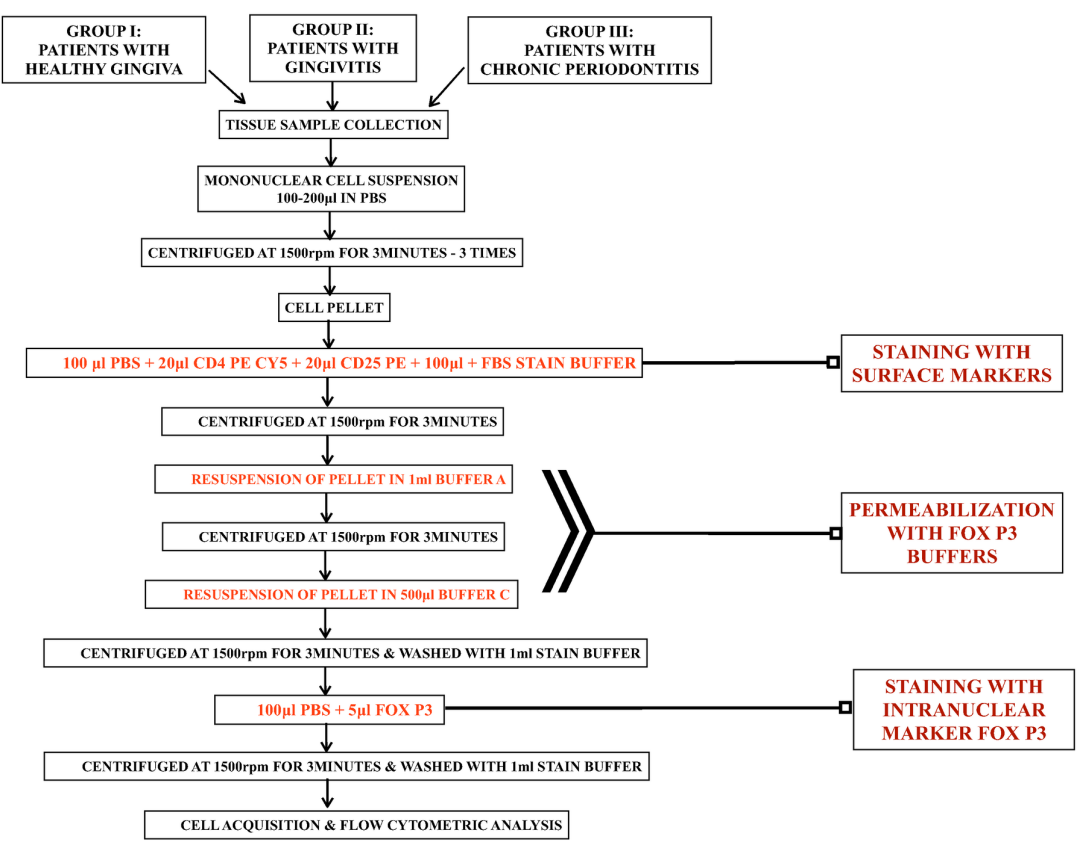

FIGURE 2: Flow diagram for tissue processing.

The gating of the cells in the gingival tissue was done for the lymphocyte region and the fluorescence of these samples was compared with the unstained sample. A total of 25,000 cells were acquired from each sample and the proportions of CD4, CD25, and Fox P3 were gated using the software.

From the gated regions, the percentage of natural induced T regulatory cells (CD4+ CD25+ FOX P3+) in the three groups (healthy, gingivitis, and chronic periodontitis) were assessed (Figure 3) and their significant differences were estimated using commercially available Statistical Package for the Social Sciences (SPSS, IBM Corp., USA) software.
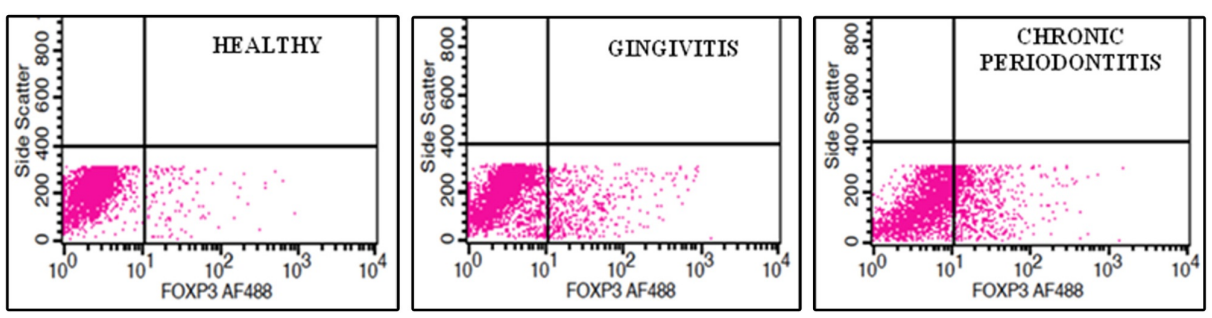

FIGURE 3: Plot diagram showing the gating of CD4+ CD25+ Fox P3+ cells in healthy, gingivitis and chronic periodontitis samples, 


\section{Cureus}

respectively.

\section{Results}

A total of 30 individuals were included in this study. They were divided into three groups based on specific inclusion and exclusion criteria as healthy individuals, patients with gingivitis, and patients with chronic periodontitis. The demographic details are summarized in Table 1 . There were four females and six males in the healthy group, three females and seven males in the gingivitis group, and two females and eight males in the chronic periodontitis group. The age range of the subjects in the control group was 21-30 years (mean age $24.5 \pm 7.74$ years), the age range of the gingivitis group was $23-35$ years (mean age $27.6 \pm 8.72$ years), and that of the chronic periodontitis group was 36-58 years (mean age $49.4 \pm 15.63$ years). The mean Simplified Oral Hygiene Index (OHI (S)) score, probing depth, and clinical attachment level (CAL) are depicted as mean \pm standard deviation in Table 1 .

\begin{tabular}{|c|c|c|c|c|}
\hline \multicolumn{2}{|l|}{ GROUP } & I HEALTHY & II GINGIVITIS & III CHRONIC PERIODONTITIS \\
\hline \multicolumn{2}{|c|}{ Number of Subjects } & 10 & 10 & 10 \\
\hline \multicolumn{2}{|c|}{ Age (in years depicted as mean \pm standard deviation) } & $24.5 \pm 7.74$ & $27.6 \pm 8.72$ & $49.4 \pm 15.63$ \\
\hline \multirow{2}{*}{ Gender } & Female & 4 & 3 & 2 \\
\hline & Male & 6 & 7 & 8 \\
\hline \multicolumn{2}{|c|}{ Mean OHI.S Score (depicted as mean \pm standard deviation) } & $0.91 \pm 0.26$ & $1.77 \pm 0.55$ & $4.69 \pm 6.95$ \\
\hline \multicolumn{2}{|c|}{ Clinical Attachment Level (depicted as mean \pm standard deviation) } & 0 & 0 & $1.98 \pm 0.62$ \\
\hline
\end{tabular}

TABLE 1: Demographic data of the study groups.

The results of our study showed an increase in the proportions of natural Tregs (CD4+ CD25+ Fox P3+) in the chronic periodontitis group compared to the gingivitis and healthy tissue groups. These values were statistically significant. However the number of natural Tregs (CD4+ CD25+ Fox P3+) was not significantly elevated in the gingivitis group compared to the healthy tissue group.

All the samples were stained initially for surface markers such as CD4 and CD25 followed by intracellular staining with Fox P3. The cells that co-expressed all the three markers (CD4, CD25, and Fox P3) were quantified as natural Tregs. ANOVA, post hoc test, and T-test were performed to analyze the results.

\section{Total CD4+ CD25+ Fox P3+ cells}

The results of our study showed an increase in the proportions of natural Tregs (CD4+ CD25+ Fox P3+) in the chronic periodontitis group compared to the gingivitis and healthy tissue groups (Figure 4). These values were statistically significant. However the number of natural Tregs (CD4+ CD25+ Fox P3+) was not significantly elevated in the gingivitis group compared to the healthy group (Tables 2-4). 


\section{Cureus}

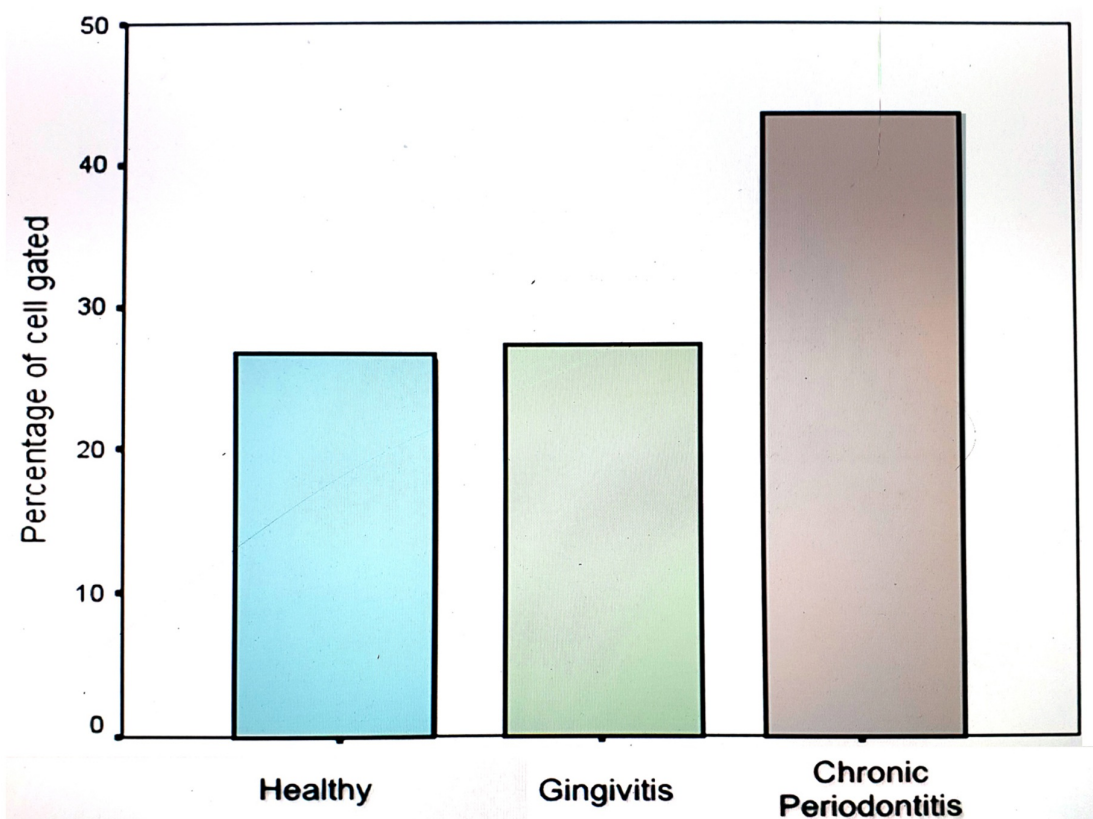

FIGURE 4: Bar graph showing the comparison of CD4+ CD25+ FOX P3+ $T$ cells in gingival tissue samples of healthy individuals, gingivitis and chronic periodontitis patients.

GROUPS

Healthy

Gingivitis

Chronic Periodontitis
$M E A N \pm$ STANDARD DEVIATION

P VALUE

$25.6171 \pm 9.30536$

$26.3900 \pm 6.48849$

$0.001^{\star *}$

TABLE 2: One way Anova Test - Comparison of gingival tissues in healthy, gingivitis, and chronic periodontitis patients for CD4+ CD25+ FoxP3+ cells

NOTE: ${ }^{\star \star}$ denotes significance: $p$ value less than 0.01 . 


\section{Cureus}

\begin{tabular}{|c|c|c|}
\hline GROUP & GROUP & SIG. \\
\hline \multirow{2}{*}{ Healthy } & Gingivitis & .982 \\
\hline & Periodontitis & $.003^{\star \star}$ \\
\hline \multirow{2}{*}{ Gingivitis } & Healthy & .982 \\
\hline & Periodontitis & $.002^{\star \star}$ \\
\hline \multirow{2}{*}{ Periodontitis } & Healthy & $.003^{\star *}$ \\
\hline & Gingivitis & $.002^{\star \star}$ \\
\hline
\end{tabular}

TABLE 3: Post hoc Tests - Tukey HSD: Multiple comparisons - Comparison of mean values of total nTreg (CD4+ CD25+ FoxP3+) cells between the groups in the gingival tissue extracts.

NOTE: *The mean difference is significant at the 0.05 level. HSD - honestly significant difference

\begin{tabular}{|c|c|c|c|c|c|c|}
\hline & GROUP & $\mathbf{N}$ & MEAN & STD. DEVIATION & STD. ERROR MEAN & P VALUE \\
\hline \multirow{5}{*}{ Percentage of cells gated } & Healthy & 10 & 26.3900 & 6.48849 & 2.45242 & \multirow{2}{*}{0.860 (NS) } \\
\hline & Gingivitis & 10 & 25.6171 & 9.30536 & 3.51710 & \\
\hline & Healthy & 10 & 26.3900 & 6.48849 & 2.45242 & \multirow{2}{*}{$0.001^{\star \star}$} \\
\hline & Periodontitis & 10 & 42.7557 & 7.59459 & 2.87049 & \\
\hline & Gingivitis & 10 & 25.6171 & 9.30536 & 3.51710 & $0.003^{\star \star}$ \\
\hline
\end{tabular}

\section{TABLE 4: T-test: Group statistics}

Comparison of mean values of total nTreg (CD4+ CD25+ FoxP3+) cells between the groups in the gingival tissue extracts.

\section{Discussion}

Periodontal disease has inflammation as the basis with many confounding factors and hence is referred to as a multifactorial disease. It is a result of host-microbial interaction where the inflammatory component leads to major destruction. Some clarity has been obtained in the pathogenesis of the disease after decades of research, but it is still far from a clear picture. An immediate acute inflammation, innate immunity, and adaptive immunity are the three different ways a host can respond [7]. A pool of undifferentiated prelymphocytes from hematopoietic stem cells after many transitions stay centrally as nTreg cells. These cells after stringent selection reach the peripheral structures. In the periphery they can differentiate into effectors factors or persist as nTregs or can be made more active induced Tregs (iTregs). Of the three, nTreg is the most immunosuppressive followed by iTreg, but the maximum tissue destruction follows effector cells [8].

In the present study we used CD4, CD25, and Fox P3 as markers to isolate nTregs from gingival tissues. Nakajima et al. in 2005 demonstrated an increase of nTregs in chronic periodontitis and gingivitis in comparison to healthy groups. An immunohistochemical analysis of CD4, CD25, and CTLA-4 and gene expression analysis of Fox P3, TGF-beta, and IL-10 in gingival biopsies revealed the presence of nTregs [9].

They suggested that nTreg cells and other regulatory $\mathrm{T}$ cell populations exist and play a role in periodontal disease. Similarly, in our present study we showed a significant increase in the nTregs in chronic periodontitis.

Cardoso et al. in their study showed similar increase in nTreg cells in chronic periodontitis and concluded that Treg cells are involved in the modulation of local immune responses in chronic lesions [10].

Ernst et al. carried out studies correlating the levels of double positive cells with 'receptor activator of nuclear factor kappa-B ligand' (RANKL) and reported that Treg cells are inversely proportional to RANKL levels depicting their role in chronic conditions [11]. Interestingly, RANKL levels are influenced by immune and inflammatory factors. So, probably, if nTregs are less, the local immune response could be more effector 
cell dominant and more destructive as evidenced by these cells.

\section{Conclusions}

The results of our study showed an increase in the proportion of nTregs (CD4+ CD25+ Fox P3+) in the chronic periodontitis group compared to the gingivitis and healthy tissue groups. These values were statistically significant. However, there was a noticeable nTreg cell expression in gingivitis that was not statistically significant. Thus, our study results conclude that nTregs play an important role in the disease progression of periodontitis.

\section{Additional Information \\ Disclosures}

Human subjects: Consent was obtained by all participants in this study. Sri Ramachandra Institute of Higher Education and Research issued approval Approved. This study was approved by the Institutional ethics committee of Sri Ramachandra University. A written informed consent was obtained from all the patients included in the study and submitted to the review board. Animal subjects: All authors have confirmed that this study did not involve animal subjects or tissue. Conflicts of interest: In compliance with the ICMJE uniform disclosure form, all authors declare the following: Payment/services info: All authors have declared that no financial support was received from any organization for the submitted work. Financial relationships: All authors have declared that they have no financial relationships at present or within the previous three years with any organizations that might have an interest in the submitted work. Other relationships: All authors have declared that there are no other relationships or activities that could appear to have influenced the submitted work.

\section{Acknowledgements}

Dr. Srujana Chitipothu (Senior Scientist, In-charge), Ms. Lakshmi Badri (Junior Scientist), Central Research Instrumentation Facility, Vision Research Foundation, Chennai, Tamil Nadu, India.

\section{References}

1. Socransky SS, Haffajee AD, Cugini MA, Smith C, Kent Jr RL: Microbial complexes in subgingival plaque. J Periodontol. 1998, 25:134-44. 10.1111/j.1600-051x.1998.tb02419.x

2. Walker LSK, Abbas AK: The enemy within: keeping self- reactive T cells at bay in the periphery reviews. Nature. 2002, 2:11-19. 10.1038/nri701

3. Buckner JH, Ziegler SF: Regulating the immune system: the induction of regulatory T cells in the periphery . J Arthritis Res Ther. 2004, 6:215-222. 10.1186/ar1226

4. Shevac EM: Certified professionals CD4+ CD25+ suppressor T cells . J Exp Med. 2001, 193:41-46.

5. Dieckmann D, Plottner H, Berchtold S, Berger T, Schuler G: Ex vivo isolation and characterization of CD4+CD25+ Tcells with regulatory properties from human blood. J Exp Med. 2001, 193:1303-1310. 10.1084/jem.193.11.1303

6. Armitage GC: Classifying periodontal diseases - a long standing dilemma . Perio 2000. 2002, 30:9-23. 10.1034/j.1600-0757.2002.03002.x

7. Kinane DF, Lappin DF: Immune process in periodontal disease: a review . Ann Periodontol. 2002, 7:62-71. 10.1902/annals.2002.7.1.62

8. Takahama Y: Journey through the thymus: stromal guides for T-cell development and selection. Nat Rev Immunol. 2006, 6:127-135. 10.1038/nri1781

9. Nakajima T, Ueki-Maruyama K, Oda T, Ohsawa Y, Ito H, Seymour GJ, Yamazaki K: Regulatory T-cells infiltrate periodontal disease tissues. J Dent Res. 2005, 84:639-643. 10.1177/154405910508400711

10. Cardoso CR, Garlet GP, Moreira AP, Júnior WM, Rossi MA, Silva JS: Characterization of CD4+CD25+ natural regulatory T cells in the inflammatory infiltrate of human chronic periodontitis. J Leukoc Biol. 2008, 84:311318. 10.1189/jlb.0108014

11. Ernst CWO, Lee JE, Nakanishi T, et al.: Diminished forkhead box P3/CD25 double-positive T regulatory cells are associated with the increased nuclear factor-kB ligand (RANKL+) T cells in bone resorption lesion of periodontal disease. J Clin Exp Immunol. 2007, 148:271-280. 10.1111/j.1365-2249.2006.03318.x 